\title{
Enseignants : exercer dans un établissement à l'étranger
}

Héloïse Nétange

\section{(2) OpenEdition}

1 Journals

Édition électronique

URL : http://journals.openedition.org/ries/867

DOI : 10.4000/ries.867

ISSN : 2261-4265

Éditeur

Centre international d'études pédagogiques

Édition imprimée

Date de publication : 1 avril 2010

Pagination : 11-14

ISSN : 1254-4590

Référence électronique

Héloïse Nétange, « Enseignants : exercer dans un établissement à l'étranger », Revue internationale

d'éducation de Sèvres [En ligne], 53 | avril 2010, mis en ligne le 01 avril 2011, consulté le 20 avril 2019.

URL : http://journals.openedition.org/ries/867 ; DOI : 10.4000/ries.867

Ce document a été généré automatiquement le 20 avril 2019

(c) Tous droits réservés 


\title{
Enseignants : exercer dans un établissement à l'étranger
}

\author{
Héloïse Nétange
}

\section{NOTE DE L'AUTEUR}

Pour faciliter la lecture, seule la racine des liens Internet est mentionnée. Pour y accéder dans leur intégralité, il suffit de consulter la rubrique Ressources en ligne sur le site de la revue : http://www.ciep.fr/sitographie .

1 Aujourd'hui, quels sont les programmes mis à la disposition des enseignants qui souhaitent partir enseigner à l'étranger? Échanges poste pour poste ou missions d'enseignement sans réciprocité, de quelques jours ou de plusieurs années scolaires, des programmes permettent aux enseignants d'exercer leur métier à l'étranger et de développer leurs compétences hors de leur contexte national, depuis l'enseignement primaire au supérieur.

2 Cette sélection vise à présenter un certain nombre de sources d'information disponibles pour les enseignants souhaitant acquérir une expérience dans un établissement à l'étranger. Elle présente des sites d'information sur les programmes européens, sur les principales possibilités de mobilité internationale en dehors du cadre de l'Union européenne et enfin elle indique les programmes nationaux ouverts aux enseignants français.

3 Sitographie arrêtée au 15 février 2010.

\section{Les programmes européens}

\section{Erasmus}

4 Le programme Erasmus est bien connu pour ses actions en faveur de la mobilité des étudiants en Europe, mais on sait moins qu'il permet également aux enseignants de 
l'enseignement supérieur de partir dispenser des cours dans un établissement européen partenaire du leur. Il s'agit de missions d'enseignement allant de cinq heures à six semaines. Le nombre de participants ne cesse de croître chaque année : 2458 enseignants français ont bénéficié de cette action au cours de l'année 2007/2008: http:// ec.europa.eu/education/programmes/llp/guide/

\section{Grundtvig}

5 L'action Grundtvig est destinée à renforcer la dimension européenne de l'éducation des adultes et à promouvoir l'éducation tout au long de la vie. Pour la formation continue du personnel de l'éducation, Gruntvig propose, outre des bourses de formation, des visites, stages d'observation et échanges destinées à tous les personnels en activité, en reconversion ou en formation (étudiants, encadrement, formateur) impliqués dans la formation des adultes. Les échanges permettent de réaliser une mission courte d'enseignement (douze semaines maximum) en Europe : http://ec.europa.eu/education/ grundtvig/doc980_en.htm

\section{Leonardo}

6 Les programmes Leonardo sont destinés à promouvoir la formation professionnelle initiale et continue au sein de l'Union européenne. Ils subventionnent des séjours d'études et des missions d'enseignement d'une à six semaines. Ces missions sont mises en place sur le principe d'un accord bilatéral entre établissements et les cours dispensés sont intégrés dans le programme officiel de l'établissement partenaire : http://ec.europa.eu/ education/leonardo-da-vinci/doc1031_en.htm

\section{L'agence Europe Education Formation France (agence 2E2F)}

7 Les enseignants français trouveront les informations relatives aux programmes européens développés ci-dessus sur le site de l'agence 2E2F, chargée de développer en France les actions du programme de formation tout au long de la vie, Lifelong Learning Program : http://www.europe-education-formation.fr

\section{Les Écoles européennes}

Les Écoles européennes (Schola Europaea) sont des établissements créés conjointement par les gouvernements des États membres de l'Union européenne et par la Communauté européenne. Elles dispensent un enseignement multilingue et multiculturel à des enfants des cycles maternel, primaire et secondaire. Elles sont destinées en priorité aux enfants des personnels des institutions européennes.

9 Les enseignants sont pour la plupart détachés pour une période déterminée par les pays membres. Pour la France, les postes vacants ou susceptibles de l'être sont publiés chaque année au bulletin officiel BO) entre le 1er mars et le 30 avril : http://www.eursc.eu/ 


\section{Quelques programmes hors Union européenne}

\section{Association universitaire de la francophonie (AUF)}

10 L'AUF propose des bourses pour des missions d'enseignement aux enseignants et aux chercheurs des 728 institutions membres de l'AUF (réparties dans 88 pays). Elles ont pour objectif de pallier un déficit passager dans une spécialité tout en favorisant les échanges entre personnels universitaires, en particulier les mobilités Sud-Sud. Pour postuler à ces missions de courte durée (de l'ordre d'une à deux semaines), les candidats doivent répondre à un appel d'offres régional d'un des neuf bureaux de l'AUF publiés dans la rubrique « appels d'offres » du site : http://www.auf.org/actions/bourse-mobilite/

\section{Programme d'échange Fulbright}

11 Créé en 1946 à l'initiative d'un sénateur américain, le programme Fulbright est un système de bourses d'études subventionné par le Département d'État des États-Unis d'Amérique et les pays partenaires. L'une de ses bourses, le Classroom Teacher exchange offre la possibilité à des enseignants américains d'échanger leur poste avec un confrère d'un des pays partenaires. Pour l'année 2010-2011, 7 pays participent à l'échange: la République tchèque, la France, la Hongrie, l'Inde, le Mexique, la Suisse et le Royaume-Uni, soit 65 postes proposés. Chaque professeur assure le service habituel de son partenaire pour un semestre ou une année scolaire : http://www.fulbrightteacherexchange.org/

\section{Programme CODOFIL}

Afin de maintenir et de développer le français en Louisiane, le programme du Conseil pour le développement du français en Louisiane (CODOFIL) et les institutions partenaires organisent un programme d'échanges de professeurs. Deux types de missions coexistent : l'enseignement du français langue seconde et le français en immersion (le français est la langue d'enseignement pour toutes les disciplines). D'une durée de trois années ces missions s'adressent à des personnes ayant déjà enseigné pendant au minimum trois ans : http://www.codofil.org/

\section{Pour les enseignants français}

13 Le ministère de l'Éducation nationale propose aux professeurs français en fonction dans les établissements publics la possibilité d'acquérir une expérience d'enseignement à l'étranger tout en conservant leur poste.

\section{Les programmes gérés par le CIEP}

14 Le Centre international d'études pédagogiques gère plusieurs programmes du ministère de l'Éducation nationale. La rubrique "Programmes de mobilité » du site du CIEP rassemble les informations sur les objectifs des programmes, les conditions de l'échange et les modalités de candidature. Il gère en particulier: les séjours professionnels pour professeur de langue vivante ou de discipline non-linguistique (DNL); le programme 
franco-louisianais CODOFIL pour la France; le programme d'échange Fulbright pour la France ; les échanges poste pour poste avec le Québec des enseignants du premier degré : www.ciep.fr

\section{Le programme Jules Verne}

Nouveau programme de mobilité internationale pour les enseignants lancé en 2009, il s'adresse à des enseignants titulaires du premier et second degré de l'enseignement public français. Trois cents postes, pour une durée d'un an reconductible une fois, ont été ouverts pour l'année scolaire 2009-2010 : www.education.gouv.fr/jules-verne

\section{Le programme d'échange franco-allemand d'enseignants du premier degré}

16 La Direction générale de l'Enseignement scolaire gère ce programme permettant aux professeurs des écoles d'enseigner le français en Allemagne pendant une année scolaire. De plus, la rubrique « Europe-International » du site Eduscol dresse la liste des dispositifs d'échange et de mobilité : http://eduscol.education.fr/

\section{Les établissements français à l'étranger}

Dans les 461 écoles, collèges et lycées français homologués par le ministère de l'Éducation nationale à travers le monde, des enfants français et étrangers suivent les programmes et cursus français.

\section{L'Agence pour l'Enseignement du Français à l'Etranger (AEFE)}

18 L'AEFE gère directement 77 établissements scolaires français à l'étranger et a passé une convention avec 166 autres établissements gérés par des associations ou des fondations, dans 130 pays. Les enseignants titulaires du ministère de l'Éducation nationale, de l'enseignement supérieur et de la recherche ayant plus de trois années d'expérience peuvent se porter candidats à des postes d'enseignement dans le primaire et le secondaire, mais aussi à des postes de direction d'écoles primaires: http:// www.aefe.diplomatie.fr/

\section{La Mission Laïque française (MLF)}

Les établissements de la Mission laïque française sont de deux types: le réseau traditionnel et les écoles d'entreprises. En tout, plus de 80 établissements à l'étranger constituent ce réseau. Plus de cinq cents postes sont ouverts chaque année à des enseignants certifiés qui peuvent justifier de trois années d'enseignement: www.mlfmonde.org 
INDEX

Index géographique : Canada, États-Unis, Union européenne

Mots-clés : enseignement, mobilité

AUTEUR

HÉLOÏSE NÉTANGE

Documentaliste au CIEP. 